\title{
Assessing the Performance of Two Bioinspired Algorithms to Solve Single-Row Layout Problem
}

\author{
Berna Haktanirlar Ulutas \\ Department of Industrial Engineering, Eskisehir Osmangazi University, 26480 Eskisehir, Turkey \\ Correspondence should be addressed to Berna Haktanirlar Ulutas; bhaktan@ogu.edu.tr
}

Received 21 March 2013; Accepted 10 October 2013

Academic Editors: A. Lockamy and G. Onwubolu

Copyright ( 2013 Berna Haktanirlar Ulutas. This is an open access article distributed under the Creative Commons Attribution License, which permits unrestricted use, distribution, and reproduction in any medium, provided the original work is properly cited.

The single-row layout problem (SRLP), also known as the one-dimensional layout problem, deals with arranging a number of rectangular machines/departments with equal or varying dimensions on a straight line. Since the problem is proved to be NP-hard, there are several heuristics developed to solve the problem. This study introduces both a Clonal Selection Algorithm (CSA) and a Bacterial Foraging Algorithm (BFA) for SRLP. The performance of the algorithms is assessed by using three (small, medium, and large sized) well known test problems available in the literature. The promising results illustrated that both algorithms had generated the best known solutions so far for most of the problems or provided better results for a number of problems.

\section{Introduction}

The objective in SRLP is to minimize the total material handling costs (MHC) and to find the optimum layout for machines in one dimension. The SRLP is also known as onedimensional layout and usually refereed as Linear Ordering Problem, where all machines have unit length. Braglia [1] pointed that the problem is widely implemented in the configuration of manufacturing systems. Kusiak and Heragu [2] stated that the type of material handling device in Flexible Manufacturing System determines the pattern to be used for the layout of machines. Therefore, the design problem that is related with material handling devices such as handling robots and Automated Guided Vehicles (AGV) is usually considered as an SRLP. Further, the problem has applications in real-life applications such as the room arrangement problem along the corridor (i.e., hotels and hospitals) [3]; the arrangement of books on a shelf in a library; the assignment of disk cylinders to files [4].

Suresh and Sahu [5] have identified the problem that has wide application areas, as an NP-complete type. Therefore, several heuristics have been proposed to solve this problem in the literature. The pioneering studies include Karp and
Held [6], Nugent et al. [7], Simmons [3], and Hall [8]. Neghabat [9] introduced a procedure where a complete solution is obtained by inserting one machine at a time to the end of the solution yet obtained. Love and Wong [10] presented a linear mixed integer-programming model for the single row layout problem and solved it using the mixed integerprogramming algorithm. Drezner [11] introduced a heuristic that is based on the eigenvectors of a transformed flow matrix. Heragu and Kusiak [12] proposed the heuristic where a pair of facilities with the largest adjusted flow is initially laid; and then the partial order is gradually completed through a loop adding new machines to the right and left of the order that is obtained in the previous iteration. Then, Heragu and Kusiak [13] introduced a linear mixed integer formulation of SRLP and solved it using a penalty method. A Simulated Annealing (SA) algorithm has been developed by Romero and Sánchez-Flores [14]. Heragu and Alfa [15] proposed a hybrid SA algorithm that combines a modified penalty algorithm and an SA to solve SRLP and multirow layout problems. Kouvelis and Chiang [16] used an SA algorithm for solving a machine layout problem with a straight line handling track. Kumar et al. [17] proposed a constructive greedy heuristic that assigns facilities with the larger number 


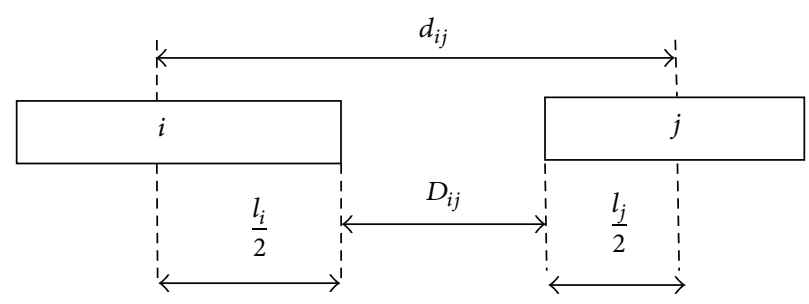

FIGURE 1: SRFLP illustration.

of moves between them. Braglia [18] proposed a combination of SA and Genetic Algorithm (GA) to minimize the total backtracking in the linear ordering of machines. The SRLP is considered with heuristics derived from scheduling problem by Braglia [1]. Ho and Moodie [19] proposed a two-phase layout procedure that combines Flow Line Analysis (FLA) and SA algorithm. Djellab and Gourgand [20] constructed an iterative procedure. Chen et al. [21] proposed an SA. Ficko et al. [22] introduced a GA. Solimanpur et al. [23] formulated the problem as a nonlinear 0-1 programming model and solved by an ant algorithm. Anjos et al. [24] constructed a semidefinite programming relaxation providing a lower bound on the optimal value of the SRLP. Amaral et al. [25] utilized SA to solve SRLP. Ponnambalam et al. [26] assessed the results of several metaheuristics with different FLA methods. Amaral [27] discussed the exact solutions for a facility layout problem. Kumar et al. [28] proposed a scatter search algorithm, Teo and Ponnambalam [29] a hybrid Ant Colony Optimisation and Particle Swarm Optimisation (ACO/PSO) algorithm, and Anjos and Vannelli [30] semidefinite programming and cutting planes. Amaral [31] introduced a new lower bound for the SRLP and Lin [32] proposed a GA. Samarghandi and Eshghi [33] considered the problem by an efficient tabu algorithm. The most recent paper of SRFLP is provided by Datta et al. [34] that utilizes a permutation-based GA.

Most of the aforementioned algorithms have rather high computational time and memory requirements for SRFLP. Boussaid et al. [35] had summarized the main metaheuristics as single solution based metaheuristics and population based. Among these known metaheuristics, this paper introduces two population based bioinspired algorithms, CSA and BFA, that are proven to generate good quality solutions for optimization problems in rather short computational times. The main aim in this paper is to assess the performance of these two novel efficient algorithms with respect to well-known test problems in the SRLP literature for the first time. The machines are assumed rectangular with different dimensions and the distance between the machines is calculated with respect to their centroids. Also, the clearance between each pair of machines is assumed as zero.

This paper is organized as follows. The second section gives brief definitions for the problem in concern. Then, the details of the algorithms are introduced in the third section. After the test problems and their sources are defined, the results available in the literature and the obtained results are compared in section four. The final section discussed the results and potential application areas for the problem.

\section{Single Row Facility Layout Problem}

SRFLP is common in manufacturing environments since some material handling equipment and machines utilize these simple and useful concepts. Figure 1 illustrates a part of a layout where $n$ machines/departments are arranged on a straight line in a given direction. The distance among the machines (i.e., $i$ and $j$ ) is defined as the sum of the center points of the machines (i.e., $l_{i} / 2$ and $l_{j} / 2$ ) and the clearance $\left(D_{i j}\right)$ between the machines, if any

$$
\begin{array}{ll}
\min \quad z=\sum_{i=1}^{n-1} \sum_{j=i+1}^{n} c_{i j} d_{i j}, \\
\text { s.t. } \quad d_{i j} \geq \frac{1}{2}\left(l_{i}+l_{j}\right)+s_{i j} ; \\
i=1,2, \ldots, n-1 ; \quad j=i+1, \ldots, n, \\
d_{i j} \geq 0 ; \quad i=1,2, \ldots, n-1 ; j=i+1, \ldots, n, \\
d_{i j}=\frac{l_{i+} l_{j}}{2}+D_{i j}, \quad D_{j i} \geq s_{i j} .
\end{array}
$$

Objective (1) is to minimize the total MHC and to find the optimum layout for machines in one dimension. Equations (2) and (3) state constraints and (4) determine the distance among two departments.

The problem is considered in several ways, such as the dimensions of the machines are either not considered or are assumed to be equal [18], the locations of facilities are predetermined $[17,18]$, and the size of the machines is only considered in the physical layout of the machines [12]. While constructing a layout, Anjos et al. [24] stated that an SRFLP with a large number of machines requires too much time. Therefore, heuristics were developed to solve the problem in a relatively short time.

\section{Bioinspired Algorithms Developed for the SRLP}

Biologically inspired computing imitates nature and uses main concepts and behavior of these systems to solve complex problems. GA is inspired from evolution, neural networks from the brain, artificial immune systems from the immune system, emergent systems from ants and bees, rendering (computer graphics) from patterning and rendering of animal skins, bird feathers, mollusk shells and bacterial colonies, and cellular automata. Along with various metaheuristics, 


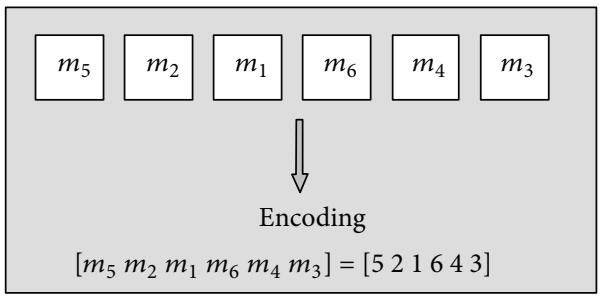

FIGURE 2: Encoding for SRFLP.

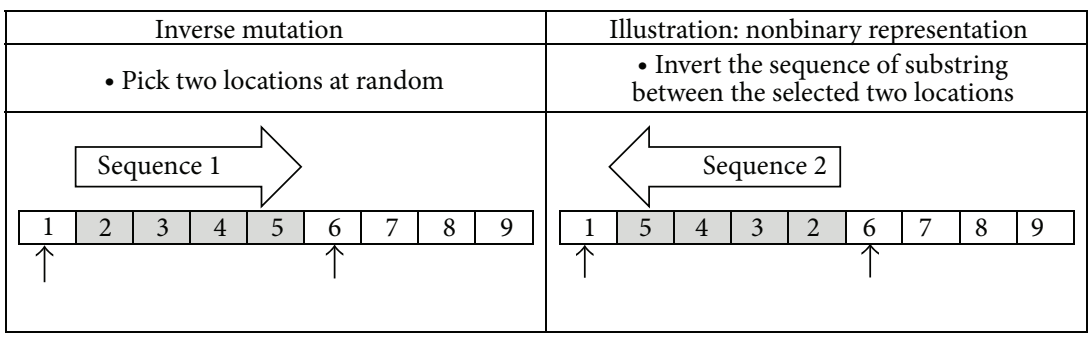

(a)

\begin{tabular}{|c|c|c|c|c|c|c|c|c|c|c|c|c|c|c|c|}
\hline \multirow{2}{*}{\multicolumn{9}{|c|}{$\begin{array}{l}\text { Pairwise-exchange (swap) mutation } \\
\text { - Pick two locations at random }\end{array}$}} & \multirow{2}{*}{\multicolumn{7}{|c|}{ Illustration: nonbinary representation }} \\
\hline & & & & & & & & & & & & & & & \\
\hline 1 & 2 & 3 & 4 & 5 & 6 & 7 & 8 & 9 & 1 & 4 & 3 & 6 & 7 & 8 & 9 \\
\hline
\end{tabular}

(b)

FIgURE 3: Two-stage mutation procedure for CSA.

the definitions and generic pseudo codes for CSA and BFA are also available in Boussaid et al. [35] for interested readers. This study that is tailored to solve SRLP provides the main algorithm steps for population generation, evaluation, reproduction, and termination of CSA and BFA.

3.1. Clonal Selection Algorithm. The clonal selection theory in an immune system is used to explain the basic response of the adaptive immune system to an antigenic stimulus. The theory depends on the idea that only cells that are capable of recognizing an antigen will proliferate [36].

The representationfor SRFLP is illustrated in Figure 2. The layout of machines in a manufacturing environment can be represented as a string array. Random layouts are generated randomly as permutations of machines.

Step 1 (initialization). The antibodies are randomly generated based on the predetermined population size.

Step 2 (evaluation). For each antibody in the population, the objective function value (MHC) is calculated.

Step 3 (selection and cloning). Selection of antibodies is made based on the MHC. The layouts that have lower MHC values have the largest share on the wheel and have more chances to be cloned.

Step 4 (hypermutation). For each antibody in the population, hypermutation operator is applied as illustrated in Figure 3. Then, objective value of the mutated antibody is calculated. If a lower MHC is obtained, the existing antibody is replaced with the mutated one. Elsewise, current antibody is kept.

Step 5 (receptor editing operator). A percentage of the antibodies (worst $R \%$ of the whole population) in the antibody population are eliminated and randomly created antibodies are replaced with them. This procedure enables the algorithm to search new regions in the solution space.

Step 6. Repeat Steps 2-5 until termination criterion is met.

In this study, the algorithm is terminated if the best feasible solution has not improved after a predetermined number of iterations (i.e., 250).

3.2. Bacterial Foraging Algorithm. Bacterial adaptability and evolvability in various habitats have attracted researchers' attention. Bacteria's behavioral strategies are then applied 
to natural computation and used to solve computational problem. In his seminal paper published in 2002, Passino pointed out how individual and groups of bacteria forage for nutrients and how to model it as a distributed optimization process, which he named the Bacterial Foraging Optimization Algorithm (BFOA). One of the major operators of BFOA is the reproduction phenomenon of virtual bacteria, each of which models one trial solution of the optimization problem.

Donangelo and Fort [37] studied the evolution of $E$. coli populations through a Bak-Sneppen-type model which incorporates random mutations. Paton et al. [38] described a bacterially-inspired computational architecture for simulating aspects of problem solving. Kim et al. [39] proposed a hybrid approach involving GA and BFA for function optimization problems and test their performance on four test functions. Datta and Misra [40] introduced an improved adaptive approach involving BFA to optimize both the amplitude and phase of the weights of a linear array of antennas. Niu et al. [41] proposed a life cycle model that is developed using an individual based modeling approach that possesses a more flexible and robust capability for simulating bacterial system compared with the population-based modeling approach. Das et al. [42] described a clustering algorithm to partite a given dataset automatically into the optimal number of groups by utilizing bacterial mutation and the gene transfer operation. Chen et al. [43] applied the cooperative approaches to the bacterial foraging optimization. Biswas et al. [44] modeled reproduction in BFA as a dynamics and analyzed the stability of the reproductive system.

Main steps of BFA to solve SRLP that is based on the foraging (i.e., searching food) strategy of E. coli bacteria are summarized as follows.

Step 1 (initialization). The bacteria are randomly generated based on the predetermined population size.

Step 2 (chemotaxis). It is a foraging strategy that implements a type of local optimization. The bacteria try to climb up the nutrient concentration, avoid noxious substances, and search for ways out of neutral media. This procedure is similar with biased random walk model.

Step 3 (Swarming). The bacteria move out from their respective places in ring of cells by moving up to the minimal value. The bacteria usually tumble, followed by another tumble or tumble followed by run or swim. As considering the case as an optimization process, the MHC that a bacterium represents is assessed. If the MHC at present is better than the cost at the previous time or duration, then the bacteria take one more step in that direction.

Step 4 (Reproduction). The bacteria are stored in ascending order based on their fitness. Then, a percent of the least healthy bacteria dies and others split into two which are placed in the same location with respect to the following steps.

(1) Nclones copies of the solution are generated so that there are $(N$ clones +1$)$ identical solutions.
(2) Inverse mutation is applied to each of the Nclones copies.

(3) The solution surviving the mutation is the nondominated solution among the mutated solutions.

(4) All other solutions are discarded.

(5) Repeat the procedure for all the solutions in the population.

Meanwhile, the population of bacteria remains constant.

Step 5. Repeat Steps 2-4 until termination criterion is met.

3.3. Algorithm Parameters. Algorithm parameter values might influence whether the algorithm will efficiently find a near-optimum solution in a reasonable time. On the other hand, choosing right parameter values is a time consuming task. Some of the studies that focus on CSA parameters are as follows. Ulutas and Islier [45] introduced a basic design of experiments only to determine CSA parameters for the equal area facility layout problems. Engin and Döyen [46] provided a generic systematic procedure that is based on a multistep experimental design approach to determine the efficient system parameters for scheduling problems. However, to the best of our knowledge, no detailed study to optimize BFA parameters is available.

3.3.1. Population Size. Since CSA and BFA are population based heuristic algorithms, population size is one of the most important parameters.

3.3.2. Elimination Ratio. The vertebrate immune system mechanism, called receptor editing, eliminates a percent of the antibodies in the CSA population. Likewise, a percent of least healthy bacteria in BFA are also discarded from the population. Parameter $R \%$ illustrates the percentage of the worst solutions to be eliminated and replaced with randomly generated ones.

3.3.3. Termination Criterion. Michalewicz [47] presented three kinds of termination conditions that have been traditionally employed. One of the following termination criteria can also be used for CSA and BFA:

(i) predetermined number of iterations,

(ii) predetermined number of objective function evaluations,

(iii) predetermined number of nonimproved iterations.

\section{Assessing the Performance of the Proposed Heuristics}

The SRLP in concern has machines with unequal dimensions (i.e., side lengths), no clearance among machines, and backtracking is not allowed. The performance of the two algorithms is tested on small and large size test problems. In this study, population size is assigned as 10 for both algorithms. Receptor editing for CSA and reproduction rate 
TABLE 1: Problem set 1: small-sized test problems with optimum solutions.

\begin{tabular}{|c|c|c|c|c|c|c|c|c|}
\hline \multirow{2}{*}{ No. } & \multicolumn{3}{|c|}{ Test problems } & \multicolumn{2}{|c|}{ Optimum solution } & \multirow{2}{*}{$\begin{array}{c}\text { Samarghandi et al. } \\
\text { [49] } \\
\text { OFV }\end{array}$} & \multirow{2}{*}{$\begin{array}{c}\text { Solimanpur et al. } \\
{[23]} \\
\text { OFV }\end{array}$} & \multirow{2}{*}{$\begin{array}{c}\text { BFA/CSA } \\
\text { OFV }\end{array}$} \\
\hline & $\begin{array}{c}\text { Problem } \\
\text { name }\end{array}$ & Reference source & $n$ & OFV & Reference & & & \\
\hline 1 & P4 & Amaral [31] & 4 & 638.00 & Amaral [27] & 638.00 & 638.00 & 638.00 \\
\hline 2 & LW5 & Love and Wong [10] & 5 & 151.00 & Amaral [27] & 151.00 & 151.00 & 151.00 \\
\hline 3 & S8_1 & Simmons [3] & 8 & 801.00 & Amaral [27] & 801.00 & $\mathrm{n} / \mathrm{a}$ & 801.00 \\
\hline 4 & S8_2 & Simmons [3] & 8 & 2324.50 & Amaral [27] & 2324.50 & $\mathrm{n} / \mathrm{a}$ & 2324.50 \\
\hline 5 & S9_1 & Simmons [3] & 9 & 2469.50 & Amaral [27] & 2469.50 & $\mathrm{n} / \mathrm{a}$ & 2469.50 \\
\hline 6 & S9_2 & Simmons [3] & 9 & 4695.50 & Amaral [27] & 4695.50 & $\mathrm{n} / \mathrm{a}$ & 4695.50 \\
\hline 7 & $\mathrm{~S} 10$ & Simmons [3] & 10 & 2781.50 & Amaral [27] & 2781.50 & $\mathrm{n} / \mathrm{a}$ & 2781.50 \\
\hline 8 & S11 & Simmons [3] & 11 & 6933.50 & Amaral [27] & 6933.50 & $\mathrm{n} / \mathrm{a}$ & 6933.50 \\
\hline 9 & LW11 & Love and Wong [10] & 11 & 6933.50 & Love and Wong [10] & 6933.50 & $\mathrm{n} / \mathrm{a}$ & 6933.50 \\
\hline 10 & P15 & Amaral [31] & 15 & 6305.00 & Amaral [27] & 6305.00 & $\mathrm{n} / \mathrm{a}$ & 6305.00 \\
\hline 11 & P17 & Amaral [31] & 17 & 9254.00 & Amaral [31] & $\mathrm{n} / \mathrm{a}$ & $\mathrm{n} / \mathrm{a}$ & 9254.00 \\
\hline 12 & P18 & Amaral [31] & 18 & 10650.50 & Amaral [31] & $\mathrm{n} / \mathrm{a}$ & $\mathrm{n} / \mathrm{a}$ & 10650.50 \\
\hline
\end{tabular}

TABle 2: Problem set 2: medium-sized test problem literature results.

\begin{tabular}{lcccccccc}
\hline \multirow{2}{*}{ No. } & \multirow{2}{*}{ Problem name } & $n$ & \multicolumn{2}{c}{ Amaral [31] } & \multicolumn{2}{c}{ Anjos and Vannelli [30] } & \multicolumn{2}{c}{ Samarghandi and Eshghi [33] } \\
& & & OFV & Time & OFV & Time & OFV \\
\hline 1 & AV25_1 & 25 & n/a & n/a & 4618.00 & 225.50 & 4631.00 & 0.04 \\
2 & AV25_2 & 25 & n/a & n/a & 37116.50 & 598.70 & 37116.50 & 0.04 \\
3 & AV25_3 & 25 & n/a & n/a & 24301.00 & 289.25 & 24560.00 & 0.05 \\
4 & AV25_4 & 25 & n/a & n/a & 48291.50 & 618.78 & 48291.50 & 0.04 \\
5 & AV25_5 & 25 & n/a & n/a & 15623.00 & 227.20 & 15623.00 & 0.04 \\
6 & AV30_1 & 30 & n/a & n/a & 8247.00 & 1540.95 & 8247.00 & 0.05 \\
7 & AV30_2 & 30 & n/a & n/a & 21582.50 & 1363.16 & 21582.50 & 0.05 \\
8 & AV30_3 & 30 & n/a & n/a & 45449.00 & 1394.13 & 46212.00 & 0.06 \\
9 & AV30_4 & 30 & n/a & n/a & 56873.50 & 8962.38 & 58297.50 & 0.06 \\
10 & AV30_5 & 30 & n/a & n/a & 115268.00 & 3936.00 & 115826.00 & 0.07 \\
11 & P35_1 & 35 & 69439.50 & n/a & n/a & n/a & 69439.50 & n/a \\
12 & P35_2 & 35 & 61712.00 & n/a & n/a & n/a & 61712.00 & n/a \\
13 & P35_3 & 35 & 69002.50 & n/a & n/a & n/a & 69002.50 & n/a \\
\hline
\end{tabular}

for BFA are considered as $10 \%$ of the population based on previous experimental studies. The first problem set given in Table 1 consists of the test problems with 4 to 18 machines that have optimum solutions.

The first column provides the information for the test problem, problem name, its reference source, and the number of machines (problem size). The optimum objective function value (OFV) for the test problems is provided in the fourth column with its reference. Following columns provide the OFVs obtained by Samarghandi and Eshghi [33] and Solimanpur et al. [23]. The last column states that BFA and CSA had generated optimum OFV for all twelve small-sized test problems. These results were obtained in less than a second for each 10 replications.
Table 2 illustrates the results gathered from the literature for twenty-two medium sized test problems. The first two columns state problem name and number of machines. Available OFVs and solution times from Amaral [31], Anjos and Vannelli [30], and Samarghandi and Eshghi [33] are given in Table 2.

Table 3 provides the results obtained from BFA are given along with the number of best results among 10 replications, best, average, and worst OFV, standard deviation (Std.Dev.) of solutions, and average of CPU seconds. The last column illustrates the improvement compared with the best known solutions. It is clear that BFA had generated all best known results available in the literature for medium sized test problems. As mentioned by Solimanpur et al. [23] and also 
TABle 3: Problem set 2: medium-sized test problem BFA results.

\begin{tabular}{|c|c|c|c|c|c|c|c|c|}
\hline \multirow{2}{*}{ Problem name } & \multirow{2}{*}{ Best known so far } & \multicolumn{7}{|c|}{ BFA } \\
\hline & & No. of best solution & Best & Average & Worst & Std.Dev. & Avg. CPU time (second) & Imp. $\%$ \\
\hline AV25_1 & 4618.00 & 9 & 4618.00 & 4618.10 & 4619.00 & 0.32 & 5.30 & 0.00 \\
\hline AV25_2 & 37116.50 & 7 & 37116.50 & 37139.00 & 37294.50 & 55.59 & 5.90 & 0.00 \\
\hline AV25_3 & 24301.00 & 10 & 24301.00 & 24301.00 & 24301.00 & 0.00 & 4.90 & 0.00 \\
\hline AV25_4 & 48291.50 & 6 & 48291.50 & 48327.20 & 48494.50 & 63.67 & 2.60 & 0.00 \\
\hline AV25_5 & 15623.00 & 8 & 15623.00 & 15630.80 & 15662.00 & 16.44 & 3.80 & 0.00 \\
\hline AV30_1 & 8247.00 & 7 & 8247.00 & 8258.90 & 8326.00 & 25.00 & 11.00 & 0.00 \\
\hline AV30_2 & 21582.50 & 4 & 21582.50 & 21609.20 & 21695.50 & 44.78 & 12.00 & 0.00 \\
\hline AV30_3 & 45449.00 & 5 & 45449.00 & 45465.70 & 45500.00 & 19.65 & 11.50 & 0.00 \\
\hline AV30_4 & 56873.50 & 7 & 56873.50 & 56884.10 & 56929.50 & 19.04 & 13.20 & 0.00 \\
\hline AV30_5 & 115268.00 & 5 & 115268.00 & 115528.60 & 116142.00 & 345.02 & 8.90 & 0.00 \\
\hline P35_1 & 69439.50 & 4 & 69439.50 & 69645.50 & 70365.50 & 346.33 & 21.50 & 0.00 \\
\hline P35_2 & 61712.00 & 1 & 61712.00 & 61819.80 & 62067.00 & 98.80 & 18.00 & 0.00 \\
\hline P35_3 & 69002.50 & 2 & 69002.50 & 69036.20 & 69092.50 & 30.27 & 14.30 & 0.00 \\
\hline
\end{tabular}

TABle 4: Problem set 2: medium-sized test problem CSA results.

\begin{tabular}{|c|c|c|c|c|c|c|c|c|}
\hline \multirow{2}{*}{ Problem name } & \multirow{2}{*}{ Best known so far } & \multicolumn{6}{|c|}{ CSA } & \multirow[b]{2}{*}{ Imp. $\%$} \\
\hline & & No. of best solution & Best & Average & Worst & Std.Dev. & Avg. CPU time (second) & \\
\hline AV25_1 & 4618.00 & 10 & 4618.00 & 4618.00 & 4618.00 & 0.00 & 2.90 & 0.00 \\
\hline AV25_2 & 37116.50 & 8 & 37116.50 & 37120.30 & 37137.50 & 8.07 & 5.40 & 0.00 \\
\hline AV25_3 & 24301.00 & 7 & 24301.00 & 24305.40 & 24333.00 & 10.01 & 3.50 & 0.00 \\
\hline AV25_4 & 48291.50 & 10 & 48291.50 & 48291.50 & 48291.50 & 0.00 & 6.50 & 0.00 \\
\hline AV25_5 & 15623.00 & 8 & 15623.00 & 15627.90 & 15662.00 & 12.39 & 3.70 & 0.00 \\
\hline AV30_1 & 8247.00 & 9 & 8247.00 & 8250.70 & 8284.00 & 11.70 & 14.90 & 0.00 \\
\hline AV30_2 & 21582.50 & 9 & 21582.50 & 21583.00 & 21587.50 & 1.58 & 14.40 & 0.00 \\
\hline AV30_3 & 45449.00 & 8 & 45449.00 & 45487.70 & 45812.00 & 114.20 & 9.70 & 0.00 \\
\hline AV30_4 & 56873.50 & 8 & 56873.50 & 56878.80 & 56901.50 & 11.20 & 12.80 & 0.00 \\
\hline AV30_5 & 115268.00 & 8 & 115268.00 & 115272.00 & 115304.00 & 11.31 & 10.40 & 0.00 \\
\hline P35_1 & 69439.50 & 6 & 69439.50 & 69480.10 & 69591.50 & 58.16 & 19.90 & 0.00 \\
\hline P35_2 & 61712.00 & 1 & 61712.00 & 61762.10 & 61811.00 & 26.14 & 38.30 & 0.00 \\
\hline P35_3 & 69002.50 & 4 & 69002.50 & 69011.30 & 69024.50 & 8.23 & 24.40 & 0.00 \\
\hline
\end{tabular}

noted by Datta et al. [34], the computational time should not be considered for comparing the performances of two algorithms as the computing machines are generally different. However, the solution times are also provided for interested readers.

Table 4 provides the problem names and best known solutions for medium sized test problems. Then, the results obtained by use of CSA are given in the following columns. Based on the column that states the best results, it is clear that CSA was able to obtain all the optimum solutions among thirteen test problems.

Since the success of the BFA and CSA are obvious for medium sized test problems considering 25, 30, and 35 machines, large sized test problems ranging from 60 up to 80 are also studied. Table 5 illustrates the twenty test problems. Available OFV and solution times from, Anjos and Yen [48], Samarghandi and Eshgi [33], and Datta et al. [34] are listed.

The solutions obtained from BFA are given in Table 6 with the number of best solutions among 10 replications, best, average, worst OFV, standard deviation, and average CPU time. The OFV generated by BFA were compared with the best known results of Datta et al. [34]. Among the twenty test problems, BFA had reached eight best known results and obtained better results for four test problems that are illustrated in bold. The rest of the eight results had generated slightly $(0.034 \%$ on the average) higher OFV.

For the P60_2 test problem, best improvement for BFA was recorded as $0.02 \%$. Ten different random seeds were used to evaluate the results generated from the algorithm. Table 7 illustrates the best results in each replication, the number of iteration and the CPU time when the best solution of the related replication was obtained, the total iteration number and total CPU time before the related iteration was terminated.

Table 8 provides CSA results for large sized test problems. The best known solutions so far are again taken from Datta et al. [34]. CSA was able to obtain the same best results for the thirteen and better results for five large sized test problems. 
TABLE 5: Problem set 3: large-sized test problem literature results.

\begin{tabular}{|c|c|c|c|c|c|c|c|c|c|c|}
\hline \multirow[b]{2}{*}{ No. } & \multirow[b]{2}{*}{$\begin{array}{l}\text { Problem } \\
\text { name }\end{array}$} & \multirow{2}{*}{$n$} & \multicolumn{2}{|c|}{ Anjos et al. [24] } & \multicolumn{2}{|c|}{ Anjos and Yen [48] } & \multicolumn{2}{|c|}{ Samarghandi et al. [49] } & \multicolumn{2}{|c|}{ Datta et al. [34] } \\
\hline & & & OFV & Time (hour) & OFV & $\begin{array}{l}\text { CPU time } \\
\text { (second) }\end{array}$ & OFV & $\begin{array}{c}\text { Time } \\
\text { (second) }\end{array}$ & OFV & $\begin{array}{c}\text { Time } \\
\text { (second) }\end{array}$ \\
\hline 1 & P60_1 & 60 & 1493704.00 & 5 & 1478464.00 & 20353 & 1477834.00 & 0.82 & 1477834.00 & 19.54 \\
\hline 2 & P60_2 & 60 & 843644.00 & 5 & 844695.00 & 18490 & 841792.00 & 0.98 & 841792.00 & 22.34 \\
\hline 3 & P60_3 & 60 & 656272.50 & 5 & 650533.50 & 17448 & 648337.00 & 0.90 & 648337.50 & 68.81 \\
\hline 4 & P60_4 & 60 & 405433.00 & 5 & 400669.00 & 17719 & 398511.00 & 0.91 & 398468.00 & 20.71 \\
\hline 5 & P60_5 & 60 & 319501.00 & 5 & 319103.00 & 18328 & 318805.00 & 0.76 & 318805.00 & 26.41 \\
\hline 6 & P70_1 & 70 & 1543098.00 & 7 & 1533075.00 & 87930 & 1529197.00 & 1.49 & 1528621.00 & 64.83 \\
\hline 7 & P70_2 & 70 & 1494182.00 & 7 & 1444720.00 & 87639 & 1441028.00 & 1.94 & 1441028.00 & 77.49 \\
\hline 8 & P70_3 & 70 & 1524171.50 & 7 & 1526830.50 & 83507 & 1518993.50 & 1.76 & 1518993.50 & 68.26 \\
\hline 9 & P70_4 & 70 & 974856.00 & 7 & 972389.00 & 82611 & 969130.00 & 1.23 & 968796.00 & 100.59 \\
\hline 10 & P70_5 & 70 & 4230912.50 & 7 & 4218730.50 & 85367 & 4218230.00 & 1.57 & 4218017.50 & 60.48 \\
\hline 11 & P75_1 & 75 & 2399583.50 & 10 & 2394812.50 & 144912 & 2393483.00 & 2.01 & 2393456.50 & 125.26 \\
\hline 12 & P75_2 & 75 & 4348544.00 & 10 & 4322967.00 & 152600 & 4321190.00 & 2.19 & 4321190.00 & 128.95 \\
\hline 13 & P75_3 & 75 & 1295085.00 & 10 & 1255634.00 & 138459 & 1248551.00 & 2.91 & 1248537.00 & 157.95 \\
\hline 14 & P75_4 & 75 & 3949276.50 & 10 & 3950444.50 & 149269 & 3942013.00 & 2.51 & 3941891.50 & 119.92 \\
\hline 15 & P75_5 & 75 & 1816455.00 & 10 & 1797676.00 & 155398 & 1791408.00 & 2.09 & 1791408.00 & 101.67 \\
\hline 16 & P80_1 & 80 & 2138083.50 & 10 & 2073453.50 & 176849 & 2069097.00 & 3.97 & 2069097.50 & 75.41 \\
\hline 17 & P80_2 & 80 & 1939938.00 & 10 & 1923506.00 & 174708 & 1921177.00 & 5.64 & 1921177.00 & 68.75 \\
\hline 18 & P80_3 & 80 & 3332421.00 & 10 & 3256577.00 & 177751 & 3251413.00 & 4.79 & 3251368.00 & 85.90 \\
\hline 19 & P80_4 & 80 & 3773429.00 & 10 & 3747950.00 & 188203 & 3746515.00 & 3.45 & 3746515.00 & 77.81 \\
\hline 20 & P80_5 & 80 & 1611495.00 & 10 & 1594228.00 & 169384 & 1589061.00 & 3.76 & 1588901.00 & 196.51 \\
\hline
\end{tabular}

TABLE 6: Problem set 3: large-sized test problem BFA results.

\begin{tabular}{|c|c|c|c|c|c|c|c|}
\hline \multirow{2}{*}{ Problem name } & \multicolumn{6}{|c|}{ BFA } & \multirow[b]{2}{*}{ Imp. $\%$} \\
\hline & No. of best solution & Best & Average & Worst & Std.Dev. & Avg. CPU & \\
\hline P60_1 & 5 & 1477834.00 & 1478543.40 & 1480894.00 & 1197.66 & 153.40 & 0.000 \\
\hline P60_2 & 2 & 841778.00 & 841952.40 & 842438.00 & 201.52 & 224.50 & 0.002 \\
\hline P60_3 & 1 & 648963.50 & 649627.30 & 650160.50 & 433.42 & 156.30 & -0.097 \\
\hline P60_4 & 1 & 398512.00 & 399606.50 & 401111.00 & 719.14 & 281.60 & -0.011 \\
\hline P60_5 & 2 & 318805.00 & 319335.40 & 321214.00 & 863.93 & 361.60 & 0.000 \\
\hline P70_1 & 1 & 1528604.00 & 1529981.90 & 1531726.00 & 980.04 & 958.00 & 0.001 \\
\hline P70_2 & 2 & 1441028.00 & 1441590.20 & 1442122.00 & 387.29 & 757.10 & 0.000 \\
\hline P70_3 & 3 & 1518993.50 & 1520008.40 & 1522350.50 & 1217.79 & 907.00 & 0.000 \\
\hline P70_4 & 2 & 968796.00 & 970542.10 & 973849.00 & 1450.53 & 1041.00 & 0.000 \\
\hline P70_5 & 1 & 4218003.00 & 4219265.30 & 4220774.50 & 1223.34 & 836.70 & 0.000 \\
\hline P75_1 & 2 & 2394527.00 & 2395851.90 & 2398617.50 & 1367.21 & 1079.40 & -0.045 \\
\hline P75_2 & 2 & 4321397.00 & 4323534.30 & 4327303.00 & 1841.17 & 1122.90 & -0.005 \\
\hline P75_3 & 1 & 1249698.00 & 1250822.50 & 1252117.00 & 872.92 & 1605.50 & -0.093 \\
\hline P75_4 & 1 & 3941816.00 & 3945090.90 & 3949730.50 & 2940.35 & 1536.50 & 0.002 \\
\hline P75_5 & 1 & 1791637.00 & 1792575.00 & 1794100.00 & 736.91 & 1652.70 & -0.013 \\
\hline P80_1 & 1 & 2069097.00 & 2070327.70 & 2071516.50 & 708.17 & 2718.00 & 0.000 \\
\hline P80_2 & 1 & 1921166.00 & 1921305.50 & 1921888.00 & 227.95 & 1916.40 & 0.001 \\
\hline P80_3 & 1 & 3251413.00 & 3254638.80 & 3257272.00 & 1813.65 & 2812.00 & -0.001 \\
\hline P80_4 & 1 & 3746712.00 & 3749103.40 & 3752445.00 & 2066.67 & 2389.20 & -0.005 \\
\hline P80_5 & 1 & 1588901.00 & 1589907.60 & 1593004.00 & 1367.44 & 2582.10 & 0.000 \\
\hline
\end{tabular}


TABLE 7: BFA results for P60_2.

\begin{tabular}{|c|c|c|c|c|c|}
\hline Replication & Best solution & Best found iteration \# & Total iteration \# & Best found time & Total time \\
\hline 1 & 841872000 & 424 & 675 & 378 & 608 \\
\hline 2 & 842122000 & 134 & 385 & 151 & 362 \\
\hline 3 & 841872000 & 65 & 316 & 70 & 282 \\
\hline 4 & 842438000 & 170 & 421 & 163 & 373 \\
\hline 5 & 841876000 & 68 & 319 & 74 & 288 \\
\hline 6 & 841778000 & 443 & 694 & 388 & 598 \\
\hline 7 & 841778000 & 533 & 784 & 471 & 685 \\
\hline 8 & 841872000 & 112 & 363 & 115 & 322 \\
\hline 9 & 841872000 & 123 & 374 & 124 & 337 \\
\hline 10 & 842044000 & 332 & 583 & 311 & 546 \\
\hline
\end{tabular}

TABLE 8: Problem set 3: large-sized test problem CSA results.

\begin{tabular}{|c|c|c|c|c|c|c|c|}
\hline \multirow{2}{*}{ Problem name } & \multicolumn{6}{|c|}{ CSA } & \multirow[b]{2}{*}{ Imp. $\%$} \\
\hline & No. of best solution & Best & Average & Worst & Std.Dev. & Avg. CPU & \\
\hline P60_1 & 9 & 1477834.00 & 1477886.00 & 1478354.00 & 164.44 & 440.70 & 0.000 \\
\hline P60_2 & 2 & 841776.00 & 841818.80 & 841872.00 & 43.33 & 504.10 & 0.002 \\
\hline P60_3 & 2 & 648337.50 & 648815.40 & 649516.50 & 356.39 & 446.70 & 0.000 \\
\hline P60_4 & 2 & 398406.00 & 398626.30 & 399281.00 & 335.25 & 469.00 & 0.016 \\
\hline P60_5 & 8 & 318805.00 & 318821.10 & 318916.00 & 36.86 & 407.80 & 0.000 \\
\hline P70_1 & 7 & 1528537.00 & 1528774.50 & 1529733.00 & 483.89 & 1587.20 & 0.005 \\
\hline P70_2 & 8 & 1441028.00 & 1441228.20 & 1443009.00 & 625.74 & 1190.60 & 0.000 \\
\hline P70_3 & 5 & 1518993.50 & 1519120.10 & 1519552.50 & 202.76 & 1394.80 & 0.000 \\
\hline P70_4 & 7 & 968796.00 & 968893.80 & 969706.00 & 285.73 & 1414.40 & 0.000 \\
\hline P70_5 & 4 & 4218048.50 & 4218460.10 & 4219044.50 & 430.83 & 1153.00 & -0.001 \\
\hline P75_1 & 1 & 2393456.50 & 2394231.40 & 2396005.50 & 1029.44 & 1739.60 & 0.000 \\
\hline P75_2 & 2 & 4321190.00 & 4321477.70 & 4322283.00 & 416.81 & 2198.60 & 0.000 \\
\hline P75_3 & 2 & 1248551.00 & 1249343.00 & 1250309.00 & 788.84 & 2062.60 & -0.001 \\
\hline P75_4 & 5 & 3941816.50 & 3942544.10 & 3944059.50 & 1003.45 & 1379.00 & 0.002 \\
\hline P75_5 & 1 & 1791408.00 & 1791884.10 & 1793044.00 & 624.40 & 2375.60 & 0.000 \\
\hline P80_1 & 2 & 2069097.50 & 2069314.70 & 2069836.50 & 273.52 & 3708.60 & 0.000 \\
\hline P80_2 & 4 & 1921136.00 & 1921170.40 & 1921212.00 & 32.80 & 2082.00 & 0.002 \\
\hline P80_3 & 3 & 3251368.00 & 3252695.90 & 3253829.00 & 1146.31 & 1987.80 & 0.000 \\
\hline P80_4 & 2 & 3746515.00 & 3747259.60 & 3750294.00 & 1247.25 & 2222.80 & 0.000 \\
\hline P80_5 & 1 & 1588901.00 & 1589531.30 & 1590593.00 & 694.64 & 2698.40 & 0.000 \\
\hline
\end{tabular}

Only two test problems were slightly worse $(0.001 \%$ on the average) than the ones provided in the literature.

For the P60_2 test problem, best improvement for CSA was recorded as $0.02 \%$. Ten different random seeds were again used to evaluate the results generated from the algorithm. Table 9 illustrates the best results in each replication, the number of iteration and the CPU time when the best solution of the related replication was obtained, the total iteration number and total CPU time before the related iteration was terminated.

Figure 4 presents the convergence graph for P60_2 the best results is obtained in the 443rd iteration by BFA and in the 137 th iteration by CSA. It is clear that CSA has a higher convergence speed than BFA.

\section{Conclusions and Results}

The single row layout problem has several applications. Although it is based on simple concepts, as the number of machines or departments increase, solution can be obtained in nonpolynomial time. Therefore, several researchers had considered the problem and provided different methods to solve the problem. This study evaluates three performance metrics such as, solution quality, speed of convergence, and frequency of hitting the optimum. CSA and BFA are used to solve the commonly studied test problems in the literature. Optimum and best known solutions were obtained for small and medium sized test problems. For the 20 large sized test problems, BFA obtained better results for the $20 \%$ and CSA 


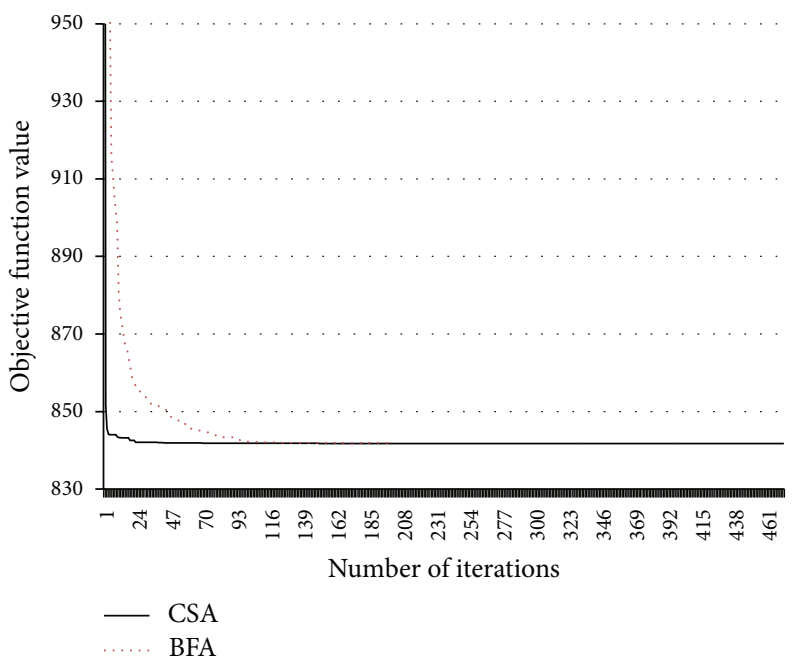

FIGURE 4: The convergence graph for P60_2 best result obtained by CSA and BFA.

TABLE 9: CSA results for P60_2.

\begin{tabular}{|c|c|c|c|c|c|}
\hline Replication & Best solution & Best found iteration \# & Total iteration \# & Best found time & Total time \\
\hline 1 & 841790000 & 382 & 633 & 904 & 1399 \\
\hline 2 & 841776000 & 137 & 388 & 343 & 856 \\
\hline 3 & 841872000 & 60 & 311 & 167 & 736 \\
\hline 4 & 841858000 & 417 & 668 & 858 & 1361 \\
\hline 5 & 841872000 & 57 & 308 & 151 & 656 \\
\hline 6 & 841872000 & 61 & 312 & 169 & 676 \\
\hline 7 & 841792000 & 300 & 551 & 708 & 1246 \\
\hline 8 & 841790000 & 199 & 450 & 441 & 941 \\
\hline 9 & 841790000 & 371 & 622 & 799 & 1297 \\
\hline 10 & 841776000 & 226 & 477 & 501 & 1075 \\
\hline
\end{tabular}

$25 \%$ of the test problems. Further, BFA and CSA obtained $40 \%$ and $65 \%$ of the best known solutions respectively. Although $40 \%$ of the solutions for the large sized test problems obtained from BFA seemed worse than literature results, the average of the improvements were calculated as $-0.034 \%$ that is very close to zero indeed. Considering the CSA solutions, only two results were worse than the best known solutions that formed the $10 \%$ of the test problems. When the results for the CSA and BFA are compared, it can be stated that both algorithms performed well for the small sized and medium sized test problems. On the other hand, CSA outperformed BFA in large sized test problems.

Further research may focus on new strategies to improve BFA search capabilities to obtain better results for layout problems. In addition, data from real life problems such as arrangement of goods in supermarkets can be obtained and the problem can be modeled as SRLP.

\section{References}

[1] M. Braglia, "Heuristics for single-row layout problems in flexible manufacturing systems," Production Planning and Control, vol. 8, no. 6, pp. 558-567, 1997.
[2] A. Kusiak and S. S. Heragu, "The facility layout problem," European Journal of Operational Research, vol. 29, no. 3, pp. 229251, 1987.

[3] D. M. Simmons, "One dimensional space allocation, an ordering algorithm," Operations Research, vol. 17, no. 5, pp. 812-826, 1969.

[4] J. C. Picard and M. Queyranne, "On the one-dimensional space allocation problem," Operations Research, vol. 29, no. 2, pp. 371391, 1981.

[5] G. Suresh and S. Sahu, "Multiobjective facility layout using simulated annealing," International Journal of Production Economics, vol. 32, no. 2, pp. 239-254, 1993.

[6] R. M. Karp and M. Held, "Finite-state process and dynamic programming," SIAM Journal of Applied Mathematics, vol. 15, pp. 201-211, 1967.

[7] C. E. Nugent, T. E. Vollman, and J. Ruml, "An experimental comparison of techniques for the assignment of facilities to locations," Operations Research, vol. 16, pp. 150-173, 1968.

[8] H. K. Hall, "An r-dimensional quadratic placement algorithm," Management Science, vol. 17, no. 3, pp. 219-229, 1970.

[9] F. Neghabat, "An efficient equipment layout algorithm," Operations Research, vol. 22, no. 3, pp. 622-628, 1974.

[10] R. F. Love and J. Y. Wong, "On solving a one-dimensional space allocation problem with integer programming," INFOR Journal, vol. 14, no. 2, pp. 139-143, 1976. 
[11] Z. Drezner, "A heuristic procedure for the layout of a large number of facilities," Management Science, vol. 33, no. 7, pp. 907915, 1987.

[12] S. S. Heragu and A. Kusiak, "Machine layout problem in flexible manufacturing systems," Operations Research, vol. 36, no. 2, pp. 258-268, 1988.

[13] S. S. Heragu and A. Kusiak, "Efficient models for the facility layout problem," European Journal of Operational Research, vol. 53, no. 1, pp. 1-13, 1991.

[14] D. Romero and A. Sánchez-Flores, "Methods for the onedimensional space allocation problem," Computers and Operations Research, vol. 17, no. 5, pp. 465-473, 1990.

[15] S. S. Heragu and A. S. Alfa, "Experimental analysis of simulated annealing based algorithms for the layout problem," European Journal of Operational Research, vol. 57, no. 2, pp. 190-202, 1992.

[16] P. Kouvelis and W. C. Chiang, "Simulated annealing procedure for single row layout problems in flexible manufacturing systems," International Journal of Production Research, vol. 30, no. 4, pp. 717-732, 1992.

[17] K. R. Kumar, G. C. Hadjinicola, and T. L. Lin, "A heuristic procedure for the single-row facility layout problem," European Journal of Operational Research, vol. 87, no. 1, pp. 65-73, 1995.

[18] M. Braglia, "Optimization of a simulated-annealing-based heuristic for single row machine layout problem by genetic algorithm," International Transactions in Operational Research, vol. 3, no. 1, pp. 37-49, 1996.

[19] Y. C. Ho and C. L. Moodie, "Machine layout with a linear singlerow flow path in an automated manufacturing system," Journal of Manufacturing Systems, vol. 17, no. 1, pp. 1-22, 1998.

[20] H. Djellab and M. Gourgand, "A new heuristic procedure for the single-row facility layout problem," International Journal of Computer Integrated Manufacturing, vol. 14, no. 3, pp. 270-280, 2001.

[21] D. S. Chen, Q. Wang, and H. C. Chen, "Linear sequencing for machine layouts by a modified simulated annealing," International Journal of Production Research, vol. 39, no. 8, pp. 17211732, 2001.

[22] M. Ficko, M. Brezocnik, and J. Balic, "Designing the layout of single- and multiple-rows flexible manufacturing system by genetic algorithms," Journal of Materials Processing Technology, vol. 157-158, pp. 150-158, 2004.

[23] M. Solimanpur, P. Vrat, and R. Shankar, "An ant algorithm for the single row layout problem in flexible manufacturing systems," Computers and Operations Research, vol. 32, no. 3, pp. 583-598, 2005.

[24] M. F. Anjos, A. Kennings, and A. Vannelli, "A semidefinite optimization approach for the single-row layout problem with unequal dimensions," Discrete Optimization, vol. 2, no. 2, pp. 113-122, 2005.

[25] A. R. S. Amaral, A. Camatta, and M. Wright, "Subset moves based simulated annealing applied to the one-dimensional layout problem," in Proceedings of the 6th Metaheuristics International Conference, Vienna, Austria, August 2005.

[26] S. G. Ponnambalam, R. Venkataraman, H. H. Sudhan, and P. V. Chatlerjee, "Hybrid search algorithms for a single-row layout in automated manufacturing systems," International Journal of Industrial Engineering, vol. 12, no. 2, pp. 117-126, 2005.

[27] A. R. S. Amaral, "On the exact solution of a facility layout problem," European Journal of Operational Research, vol. 173, no. 2, pp. 508-518, 2006.
[28] S. Kumar, P. Asokan, S. Kumanan, and B. Varma, "Scatter search algorithm for single row layout problem in FMS," Advances in Production Engineering and Management, vol. 3, no. 4, pp. 193204, 2008.

[29] Y. T. Teo and S. G. Ponnambalam, "A hybrid ACO/PSO heuristic to solve single row layout problem," in Proceedings of the 4th IEEE Conference on Automation Science and Engineering (CASE '08), pp. 597-602, Washington, DC, USA, August 2008.

[30] M. F. Anjos and A. Vannelli, "Computing globally optimal solutions for single-row layout problems using semidefinite programming and cutting planes," INFORMS Journal on Computing, vol. 20, no. 4, pp. 611-617, 2008.

[31] A. R. S. Amaral, "A new lower bound for the single row facility layout problem," Discrete Applied Mathematics, vol. 157, no. 1, pp. 183-190, 2009.

[32] M. T. Lin, "The single-row machine layout problem in apparel manufacturing by hierarchical order-based genetic algorithm," International Journal of Clothing Science and Technology, vol. 21, no. 1, pp. 31-43, 2009.

[33] H. Samarghandi and K. Eshghi, "An efficient tabu algorithm for the single row facility layout problem," European Journal of Operational Research, vol. 205, no. 1, pp. 98-105, 2010.

[34] D. Datta, A. R. S. Amaral, and J. R. Figueira, "Single row facility layout problem using a permutation-based genetic algorithm," European Journal of Operational Research, vol. 213, no. 2, pp. 388-394, 2011.

[35] I. Boussaid, J. Lepagnot, and P. Siarry, "A survey on optimization metaheuristics," Information Sciences, vol. 237, pp. 82-117, 2013.

[36] F. M. Burnet, The Clonal Selection Theory of Acquired Immunity, Cambridge University Press, Cambridge, UK, 1959.

[37] R. H. Donangelo and H. Fort, "Model for mutation in bacterial populations," Physical Review Letters, vol. 89, no. 3, Article ID 038101, 4 pages, 2002.

[38] R. C. Paton, C. Vlachos, Q. H. Wu, and J. R. Saunders, "Simulated bacterially-inspired problem solving-the behavioural domain," Natural Computing, vol. 5, no. 1, pp. 43-65, 2006.

[39] D. H. Kim, A. Abraham, and J. H. Cho, "A hybrid genetic algorithm and bacterial foraging approach for global optimization," Information Sciences, vol. 177, no. 18, pp. 3918-3937, 2007.

[40] T. Datta and I. S. Misra, "Improved adaptive bacteria foraging algorithm in optimization of antenna array for faster convergence," Progress in Electromagnetics Research C, vol. 1, pp. 143157, 2008.

[41] B. Niu, Y. L. Zhu, X. X. He, H. Shen, and Q. H. Wu, "A lifecycle model for simulating bacterial evolution," Neurocomputing, vol. 72, no. 1-3, pp. 142-148, 2008.

[42] S. Das, A. Chowdhury, and A. Abraham, "A bacterial evolutionary algorithm for automatic data clustering," in Proceedings of the IEEE Congress on Evolutionary Computation (CEC '09), pp. 2403-2410, Trondheim, Norway, May 2009.

[43] H. Chen, Y. Zhu, and K. Hu, "Cooperative bacterial foraging optimization," Discrete Dynamics in Nature and Society, vol. 2009, Article ID 815247, 17 pages, 2009.

[44] A. Biswas, S. Das, A. Abraham, and S. Dasgupta, "Stability analysis of the reproduction operator in bacterial foraging optimization," Theoretical Computer Science, vol. 411, no. 21, pp. 2127-2139, 2010.

[45] B. H. Ulutas and A. A. Islier, "Parameter setting for clonal selection algorithm in facility layout problems," in Computational Science and Its Applications-ICCSA 2007, O. Gervasi and M. Gavrilova, Eds., vol. 4705 of Lecture Notes in Computer Science, part 1, pp. 886-899, Springer, Berlin, Germany, 2007. 
[46] O. Engin and A. Döyen, "A new approach to solve hybrid flow shop scheduling problems by artificial immune system," Future Generation Computer Systems, vol. 20, no. 6, pp. 1083-1095, 2004.

[47] Z. Michalewicz, Genetic Algorithms + Data Structures = Evolution Programs, Springer, New York, NY, USA, 1996.

[48] M. F. Anjos and G. Yen, "Provably near-optimal solutions for very large single-row facility layout problems," Optimization Methods and Software, vol. 24, no. 4-5, pp. 805-817, 2009.

[49] H. Samarghandi, P. Taabayan, and F. F. Jahantigh, "A particle swarm optimization for the single row facility layout problem," Computers and Industrial Engineering, vol. 58, no. 4, pp. 529534, 2010. 

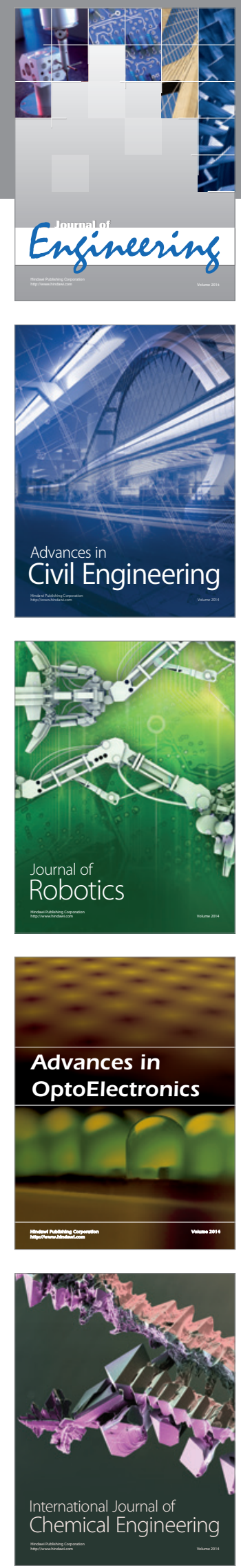

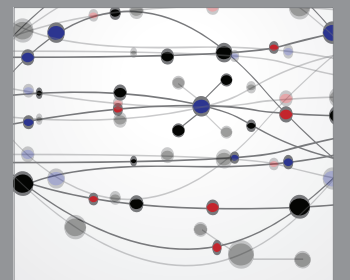

The Scientific World Journal
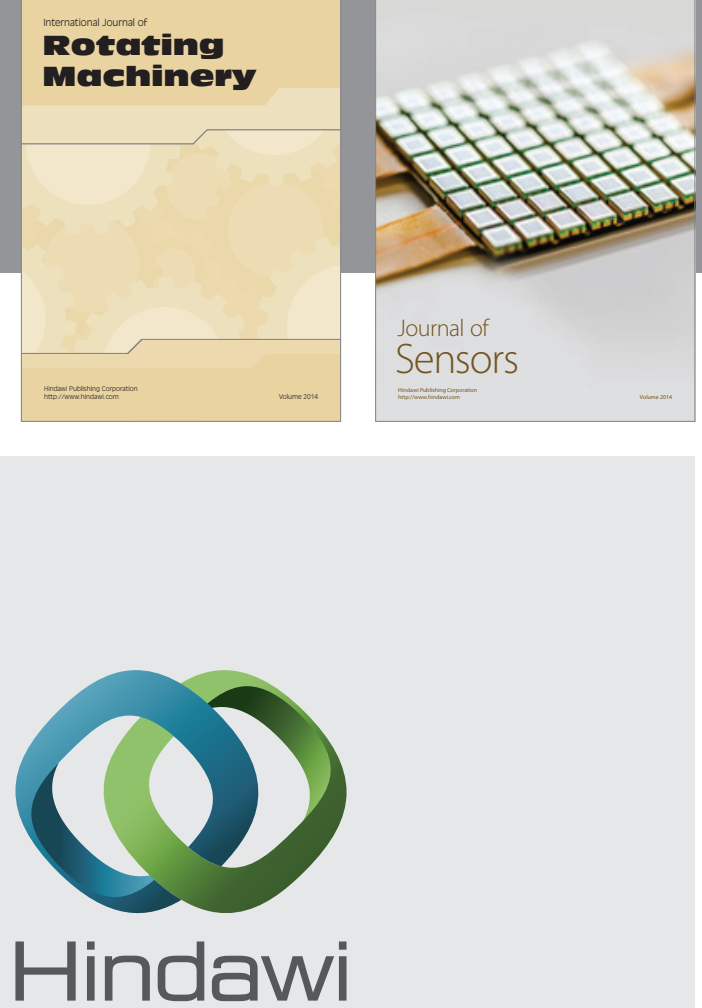

Submit your manuscripts at http://www.hindawi.com
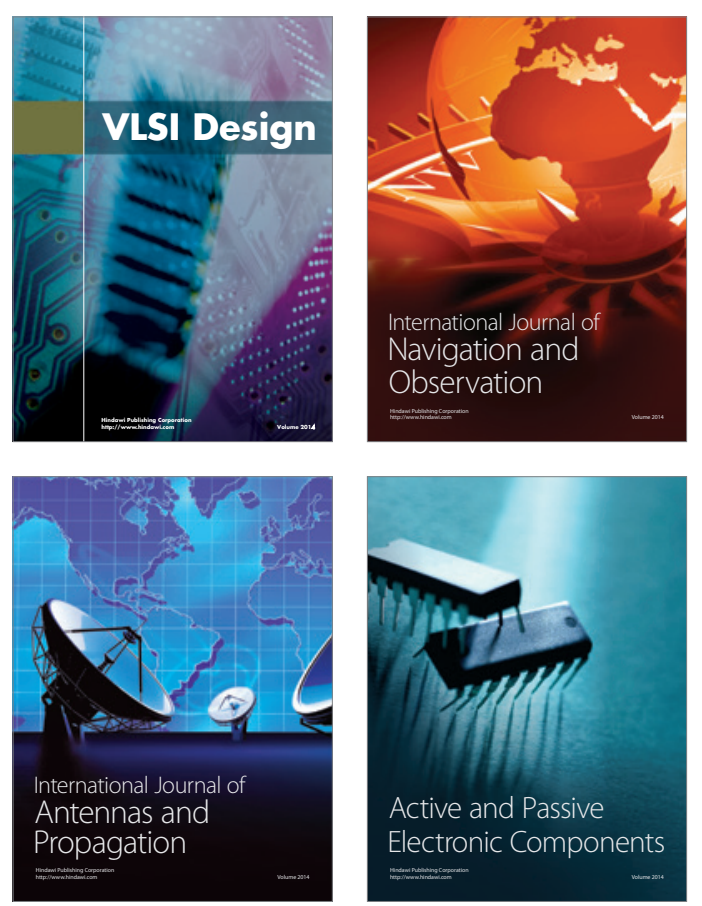
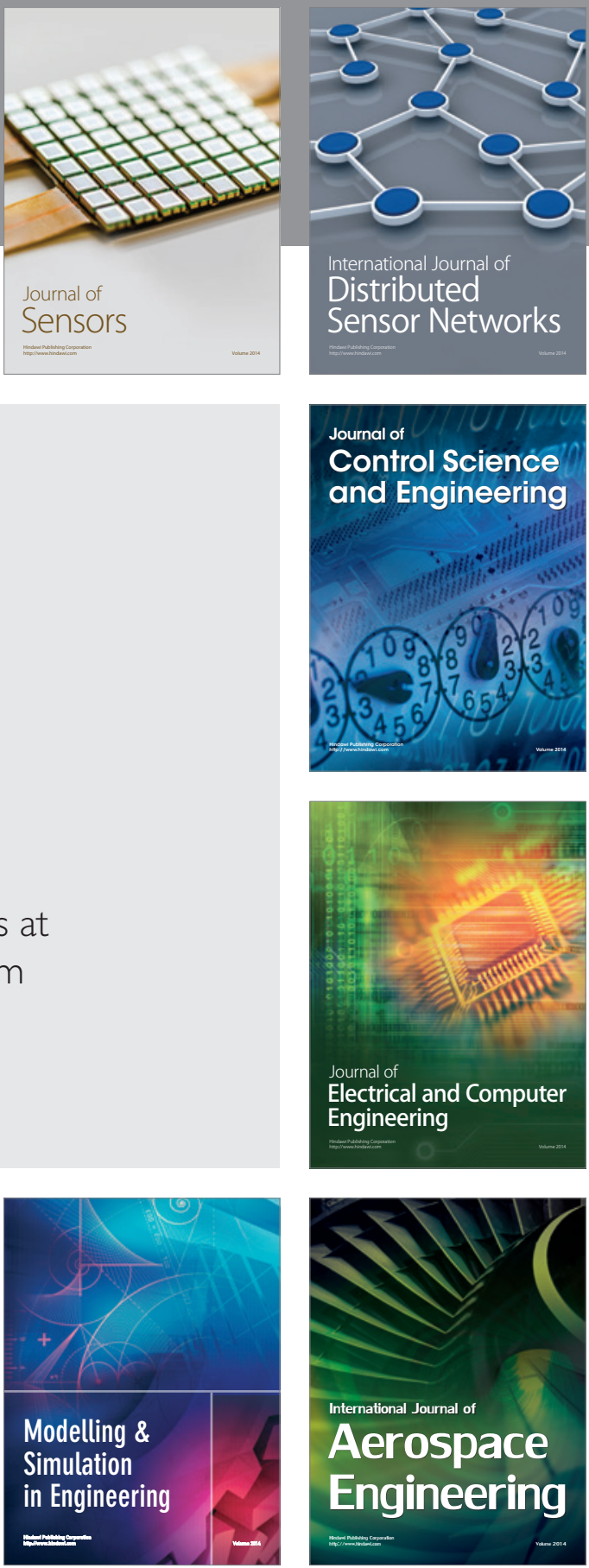

Journal of

Control Science

and Engineering
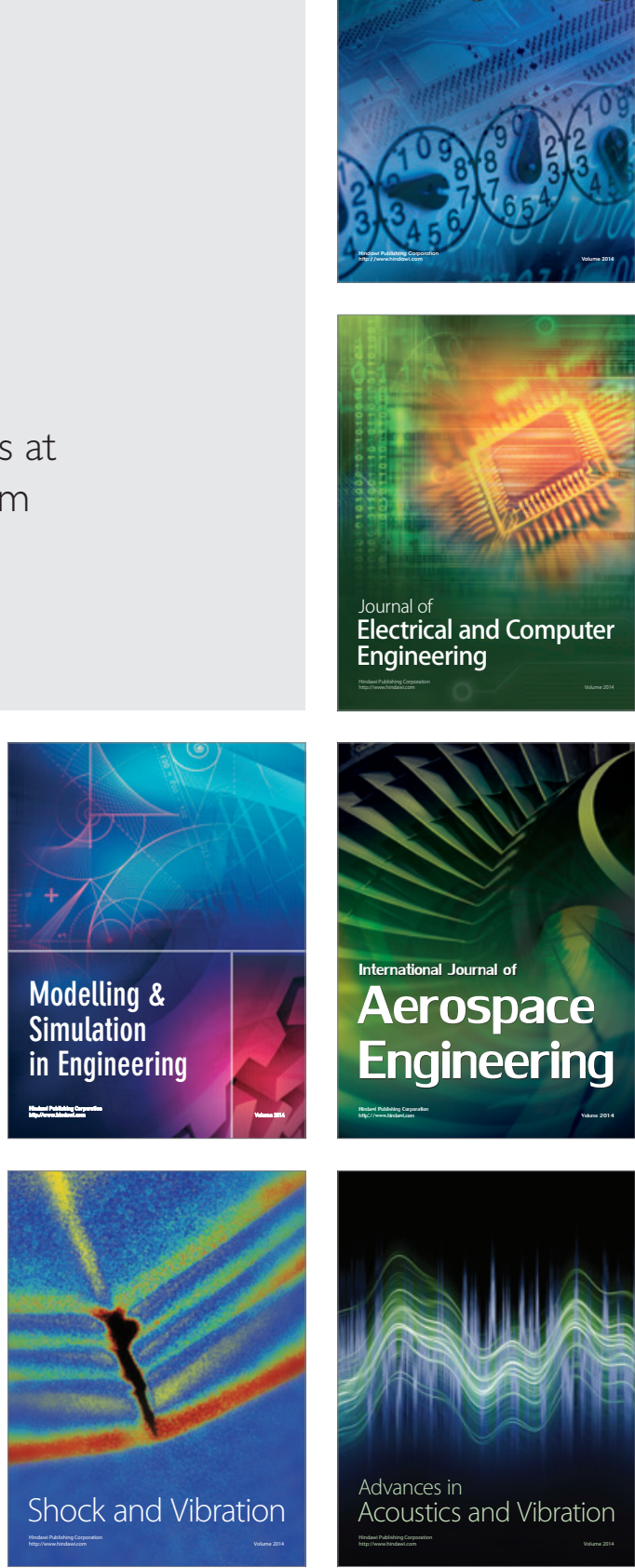